\title{
Application of Different Models for the Prediction of the Kinetics of Direct Reduction of Natural Iron Ores
}

\author{
Abraham J. B. Muwanguzi ${ }^{*}$, Aliaksandr Alevanau1, Joseph K. Byaruhanga² \\ ${ }^{1}$ Department of Material Science and Engineering, KTH Royal Institute of Technology, Stockholm, Sweden \\ ${ }^{2}$ Department of Mechanical Engineering, School of Engineering, College of Engineering Design Art and Technology, Makerere \\ University, Kampala, Uganda \\ Email: *ajbm@kth.se, *ajbmuwanguzi@gmail.com
}

How to cite this paper: Muwanguzi, A.J.B., Alevanau, A. and Byaruhanga, J.K. (2017) Application of Different Models for the Prediction of the Kinetics of Direct Reduction of Natural Iron Ores. Geomaterials, 7, 117-131.

https://doi.org/10.4236/gm.2017.74009

Received: September 11, 2017

Accepted: October 22, 2017

Published: October 25, 2017

Copyright $\odot 2017$ by authors and Scientific Research Publishing Inc. This work is licensed under the Creative Commons Attribution International License (CC BY 4.0).

http://creativecommons.org/licenses/by/4.0/

\begin{abstract}
Simulation of the direct reduction conditions was performed in a laboratory furnace. Lump samples from natural hematite iron ore were reduced by a gas mixture of $\mathrm{H}_{2}$ and $\mathrm{CO}\left(\mathrm{H}_{2} / \mathrm{CO}=1.5\right)$ at temperatures of $700^{\circ} \mathrm{C}, 800^{\circ} \mathrm{C}$ and $900^{\circ} \mathrm{C}$. The effect of reduction temperature on the reduction degree, reduction rate of samples and carbon deposition were investigated and discussed in this study. The thermo-gravimetric data obtained from the reduction experiments was run in a programme that calculates the solid conversion rate. Also, three models: 1) Grain Model (GM), 2) Volumetric Model (VM), and 3) the Random Pore Model (RPM), were used to estimate the reduction kinetics of natural iron ores. It was found that the RPM model result agreed best with the obtained experimental results. Furthermore, it gave better predictions of the natural iron oxide conversion and thereby the reduction kinetics.
\end{abstract}

\section{Keywords}

Iron Ore, Reduction Kinetics, Solid Conversion, Reduction Rate, DR Estimation Models

\section{Introduction}

In the direct reduction of iron oxide with gas as the reducing agent, a reduction reaction takes place. First, at the initial point of contact (chemical reaction) and then within the core as the gas penetrates the lump iron oxide (diffusion). The two mechanisms, together with the mass transfer of reactant products through the reduced mass, take place simultaneously. Understanding the dynamics of these mechanisms for a given iron oxide is a crucial factor in setting the reduc- 
tion conditions to achieve the desired reduction degree (RD). A number of parameters influence the reduction dynamics ranging from the iron oxide properties to the reducing conditions that exist within the shaft of the furnace at a given time. Studies modeling the reduction process to get a good understanding of the direct reduction dynamics have been conducted: 1) within a shaft reactor [1] [2] [3] [4];2) for iron oxide pellets and sinters reacting with carbon monoxide or hydrogen separately [1] [2] and as gas mixtures [3] [4].

Reduction takes place as the reducing gas penetrates through the pores of the iron oxide and the mode of reduction will vary depending on the porosity. The larger the pore surface area, the smaller is the fraction of the total pore surface walls utilized in the reaction [4]. As reduction proceeds, the mean pore diameter increases. This means that some pores grow bigger in size and others are destroyed as reduction proceeds. Furthermore, for the case of $\mathrm{CO}$ as the reducing gas, the higher the concentration, the larger the resultant pore diameter [3]; this implies increased reduction.

In studying the characteristics of pores of hematite iron reduced in hydrogen, Turkdogan and Vinters [2] noted that most of the pores in the reduced iron were interconnected. This may imply that $\mathrm{H}_{2}$ gas penetrates the hematite core more than $\mathrm{CO}$. This may explain the use of these two gases $\left(\mathrm{H}_{2} / \mathrm{CO}\right)$ in combination during the reduction process. Though both have varying reduction potentials at different temperatures, it seems that $\mathrm{H}_{2}$ creates passages in the iron ore core, which $\mathrm{CO}$ uses to reduce the core as reduction progresses.

Furthermore, that the pore area increased with a decreasing reduction temperature indicates a slowdown in the reduction process. The pore structure of the reduced iron becomes finer with a decreasing reduction temperature, leading to a decreased pore size, an increased pore surface area, and a decreased effective gas diffusivity [4].

At the on-set of the hematite reduction, the gas-solid topochemical reactions produce a layer of metallic iron at the surface in contact with a wustite layer below it. Below the wustite layer, a magnetite layer surrounding a hematite core can be found. As the reduction progresses, the reducing gas thus has to diffuse through these layers as hematite is being reduced to its lower oxides. Various models have been developed to describe the reduction dynamics within the iron oxide [5] [6] [7].

The grain model (GM) [5] assumes that solid conversion takes place around each grain within the sample. Also, that the reduction gas penetrates the pores of the solid and reduces the sample on the surface of each individual grain. The grains are assumed to be spherical, uniform and non-porous. The shrinking core phenomenon is thus applied on each individual grain. The volumetric model (VM) [6] considers the sample as a whole and assumes a homogenous conversion with a linearly decreasing reaction surface. The VM model does not account for the porous nature of the solid during the reduction process. The random pore model (RPM) [7] takes the overlapping of the pores within the solid into consideration. On this account, there is thus a total reduced pore area for gas 
penetration during the process of reduction. The model considers the effect of pore growth during the initial stages of reduction and the destruction of neighboring pores due to the overlapping during the reduction process.

None of these models may exclusively stand alone to fully describe the reduction kinetics. The GM model can describe reduction on a micro level within the oxide, but may fall short in giving a wholesome description of the reduction behavior. Grains also have various shapes and porosity. Thus, considering them to be homogenous may not give accurate results. However, it may predict the reduction kinetics of very porous iron oxides with a good degree of accuracy. The VM model may predict the reduction behavior of dense iron oxides more accurately, though in reality, there is no iron oxide that is completely without pores. The GM model and VM model may work well at predicting reduction dynamics of sinters and pellets whose physical properties can be controlled and determined during the process of their production. The RPM model gives a harmonized prediction between the above two models and may be closer to predicting the reduction kinetics of natural iron oxides.

In this study, the three models were applied to the experimental data of the reduced natural iron ore to identify which one provides a more accurate prediction of the natural iron ore conversion. With the identified model, the kinetics of direct reduction of the iron oxide can then be studied and understood. Since most of the previous studies have been carried out on sinters and pellets, the identified model can be used to study the kinetics of direct reduction of other naturally occurring iron ores.

\section{Materials and Methods}

\subsection{Materials}

A lump natural hematite iron ore from Muko deposit (Uganda), chemical composition given in Table 1, was crushed. The resulting sample pieces, with varying weights, were directly reduced in a reduction furnace. A mixture of $\mathrm{H}_{2} / \mathrm{CO}$ $(=1.5)$ was used as the reducing gas, at different flow rates with three reduction temperatures: $700^{\circ} \mathrm{C}, 800^{\circ} \mathrm{C}$ and $900^{\circ} \mathrm{C}$.

\subsection{Methods}

A schematic diagram of the thermo-gravimetric reduction equipment used in the experiments is shown in Figure 1. The detailed reduction procedure is described by Muwanguzi et al. [8].

The reduction degree of iron ore (RD) achieved for the various samples was calculated using the following equation:

Table 1. Chemical composition of hematite iron ore used for reduction experiments.

\begin{tabular}{ccccccccccc}
\hline \multicolumn{10}{c}{ Chemical composition (wt \%) } \\
\hline $\mathrm{Fe}_{2} \mathrm{O}_{3}$ & $\mathrm{FeO}$ & $\mathrm{SiO}_{2}$ & $\mathrm{Al}_{2} \mathrm{O}_{3}$ & $\mathrm{MnO}$ & $\mathrm{TiO}_{2}$ & $\mathrm{ZnO}$ & $\mathrm{CuO}$ & $\mathrm{MgO}$ & $\mathrm{P}_{2} \mathrm{O}_{5}$ & $\mathrm{~S}$ \\
\hline 92.6 & 0.44 & 2.28 & 1.35 & 0.01 & 0.07 & $<0.01$ & $<0.01$ & 0.11 & 0.02 & 0.001 \\
\hline
\end{tabular}




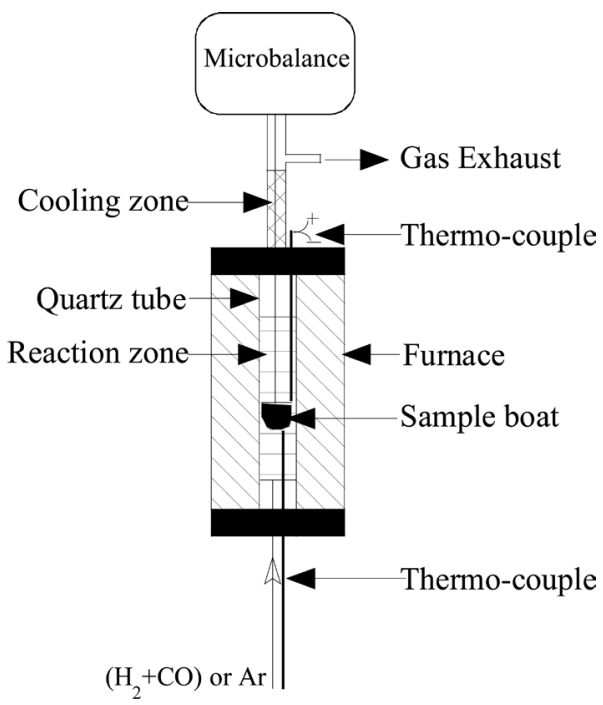

Figure 1. Schematic diagram of the equipment for the reduction experiments.

$$
\mathrm{RD}=\frac{\Delta W}{W_{0} \cdot \% \mathrm{O} / 100 \%}=100 \% \cdot \frac{W_{0}-W}{\left[W_{0} \cdot\left(\% \mathrm{Fe}_{2} \mathrm{O}_{3} \cdot 48 / 160+\% \mathrm{FeO} \cdot 16 / 72\right)\right]}
$$

where $\Delta W\left(=W_{0}-W\right)$ is the difference in sample weight before and after reduction, which corresponds to the $\mathrm{O}$ weight reduced during an experiment. The parameters $W_{0}$ and $W$ are the weights of a sample before and after a reduction experiment. The parameter $\% \mathrm{O}$ is the weight percentage of oxygen that can be removed from the $\mathrm{Fe}_{2} \mathrm{O}_{3}$ and $\mathrm{FeO}$ in a sample. Finally, the parameters $\% \mathrm{Fe}_{2} \mathrm{O}_{3}$ and $\% \mathrm{FeO}$ are the weight percentages of respective oxides in the natural iron ore.

\subsection{Description of Models Used}

The reduction experimental data was run in a programme that calculates the solid conversion rate and fits this with three different models (the grain model (GM) [5], the volumetric model (VM) [6] and the random pore model (RPM) [7]) that estimate the kinetics of reduction. All the three models were implemented in a Scilab program using the algorithm based on a least square optimization procedure. It analyses all the kinetic parameters of the models to achieve the best fits of the obtained experimental data at every temperature. Independent variation of every kinetic parameter (frequency and form factors, activation energy and parameter $\Psi$ ) allows test of compliance of the apparent conversion kinetics to the Arrhenius law. A strict compliance to this law has to give the same kinetic parameters for the best fits at every temperature. However, because the actual kinetics of the reduction process depend on such factors as deposition of carbon on dynamically changing pores (this dynamics in turn is also temperature dependent), the exact compliance of apparent kinetics to Arrhenius law may not be achieved. This is apparent from the analysis of the kinetic parameters obtained by the algorithm as will be discussed later.

The general kinetics expression of the overall solid conversion rate is as given 
in Equation (2).

$$
\frac{\mathrm{d} X}{\mathrm{~d} t}=k\left(C_{g}, T\right) f(X)
$$

where $k$ is the apparent reduction rate, and it takes into account the effects of the reducing gas concentration, $C_{g}$ and the reducing temperature, $T$. The changes in the physical and chemical properties of the samples are taken into consideration by the factor, $f(X)$. The parameter $X$ is the extent of conversion during the reduction experiment and $t$ is the reduction time. The apparent reduction rate, which is dependent on temperature, can be expressed using the Arrhenius Equation (3):

$$
k=k_{0} \mathrm{e}^{\frac{-E_{a}}{R T}}
$$

where $k_{0}$ and $E_{a}$ are the pre-exponential factor and activation energy, respectively. $R$ is the universal gas constant $(=8.314 \mathrm{~J} / \mathrm{mol} \mathrm{K})$.

To describe the kinetics of reduction of the natural iron ore, three different models where used, which give a different formulation of the function $f(X)$. With the GM model [5], the overall reaction rate is expressed as illustrated in Equation (4):

$$
\frac{\mathrm{d} X}{\mathrm{~d} t}=k_{\mathrm{GM}}(1-X)^{2 / 3}
$$

The VM model [6] expresses the overall reaction rate as shown in Equation (5):

$$
\frac{\mathrm{d} X}{\mathrm{~d} t}=k_{\mathrm{VM}}(1-X)
$$

Furthermore, for the RPM model [7], the overall reaction rate can be calculated as follows:

$$
\frac{\mathrm{d} X}{\mathrm{~d} t}=k_{\mathrm{RPM}}(1-X) \sqrt{[1-\Psi \ln (1-X)]}
$$

The RPM model introduces the factor, $\Psi$, which is the structural parameter related to the pore surface of unreacted sample $(X=0)$. This can be described as in Equation (7):

$$
\Psi=\frac{4 \pi L_{0}\left(1-\varepsilon_{0}\right)}{S_{0}^{2}}
$$

where $L_{0}, \varepsilon_{0}$ and $S_{0}$ are the pore length, solid porosity and pore surface area, respectively.

The linearized Equations of (4)-(6) are as highlighted in Equations (8)-(10) respectively:

$$
\begin{gathered}
k_{\mathrm{GM}} t=3\left[1-(1-X)^{1 / 3}\right] \\
k_{\mathrm{VM}} t=-\ln (1-X) \\
k_{\mathrm{RPM}} t=(2 / \Psi)[\sqrt{[1-\Psi \ln (1-X)]}-1]
\end{gathered}
$$


The three models were applied to the data of thermo-gravimetric experiments on raw iron ore conversion, and the conversion predictions made by each model are further discussed.

\section{Results and Discussion}

Figure 2 illustrates the reduction rates (RR) of the hematite iron oxide samples obtained during reduction experiments at a given gas flow rate $(0.5 \mathrm{~L} / \mathrm{min})$ and for different temperatures $\left(700^{\circ} \mathrm{C}, 800^{\circ} \mathrm{C}\right.$ and $\left.900^{\circ} \mathrm{C}\right)$. The $\mathrm{RR}$ values for given $i$-th time step $\left(\Delta \tau_{i}\right)$ were calculated from the experimental data as follows:

$$
\mathrm{RR}=\Delta W_{i} / \Delta \tau_{i}
$$

where $\Delta W_{i}$ is the change of sample weight during the $i$-th time step.

It can be seen in Figure 2 that the reduction rate at all temperatures increased with time and reached the maximum values after 200 - 300 seconds (Period I). During this period, chemical reactions on the surface of the iron oxide provided the higher reduction rate. Moreover, a penetration of reduction gas into the pores in natural ore lumps also contributed to the increasing of the reduction rate. The highest $R R$ value $(1.08 \mathrm{mg} / \mathrm{s})$ was achieved in the reduction experiment at a $900^{\circ} \mathrm{C}$ temperature. The maximum values of reduction rate decrease significantly with a decreased reduction temperature (till 0.85 and $0.75 \mathrm{mg} / \mathrm{s}$ for 800 and $700^{\circ} \mathrm{C}$, respectively). This is due to the decreased rate of chemical reactions. Furthermore, the size of pores, which are formed during reduction of the iron oxides, and gas diffusion are decreased with the decreasing temperature [4].

After 200 - 300 seconds, the RR values in all the reduction experiments started to fast decrease almost linearly during the following 500 - 1500 seconds (Period II). It can be explained by the increased resistance to reduction gas diffusion

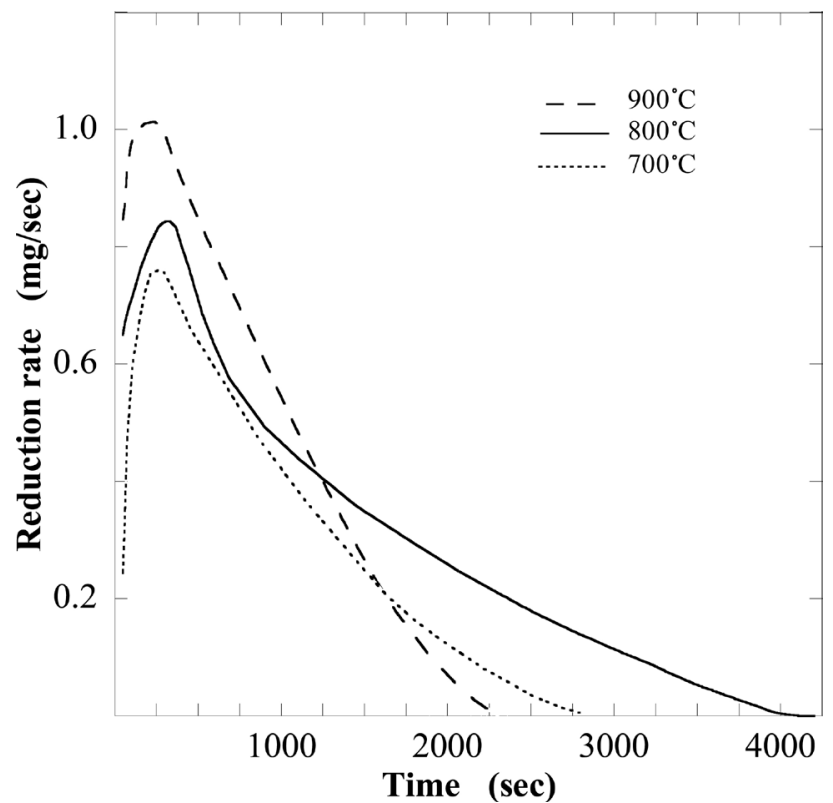

Figure 2. Reduction rates of natural iron ore as a function of temperature, calculated from experimental data at a flow rate of $0.5 \mathrm{~L} / \mathrm{min}$. 
through the reduced iron layer. It is clear that the resistance to the diffusion of reduction gas into the iron oxide lump is greatly increased due to the increased thickness of the reduced iron layer during the reduction experiment. The typical compositions of the different layers in hematite iron ore sample reduced at a $900^{\circ} \mathrm{C}$ temperature and $0.5 \mathrm{~L} / \mathrm{min}$ of gas flow rate are given in Table 2 . It can be seen that the significantly higher iron content $(95 \%-99 \%$ in Layer 1) was obtained near the sample surface than at the centre $(70 \%-77 \%$ in Layer 3$)$. According to obtained contents of $\mathrm{Fe}$ and $\mathrm{O}$ in respective zones, it is believed that Layer 1 in a lump surface after reduction experiments consists mostly of reduced Fe and 5\% - 22\% of FeO. Also, Layer 2 contains mostly $\mathrm{FeO}(60 \%-90 \%)$ and reduced $\mathrm{Fe}(40 \%-10 \%)$. Layer 3 represents a mixture of $\mathrm{FeO}, \mathrm{Fe}_{3} \mathrm{O}_{4}$ and $\mathrm{Fe}_{2} \mathrm{O}_{3}$. It was found that the thickness of these layers was varied in wide ranges and it depended on the reduction temperature, original size and microstructure (porosity and etc.) of the iron ore lumps.

In the final period (Period III), Figure 2, the reduction rate was in general controlled by the diffusion process into the grain volume through the reduced iron layers. In this case, the RR value is relatively small. It is apparent that the contributions of the reduction processes on the outside surface layer of the sample $\left(R_{\mathrm{OS}}\right)$, on the inside surface of the open pores $\left(\mathrm{RR}_{\mathrm{IS}}\right)$ of the sample and in the volume of the iron oxide grains $\left(\mathrm{RR}_{\mathrm{VG}}\right)$ to the total reduction rate $(\mathrm{RR})$ are different at the different periods of the reduction experiments.

Moreover, the deposition of carbon on a surface of the sample during reduction experiments can significantly decrease the reduction rate and reduction degree. Nouri et al. [9] reported that the carbon deposition increases with a decreasing reduction temperature during the reduction of iron oxide with $\mathrm{CO}$. This phenomenon was also observed in this study. It was found that the carbon deposition increased on average from $0.54 \%$ at $900^{\circ} \mathrm{C}$ and 1.32 at $800^{\circ} \mathrm{C}$ to $6.42 \%$ at $700^{\circ} \mathrm{C}$, as illustrated in Figure 3. Some dispersion of obtained results in the experiments at $800^{\circ} \mathrm{C}$ and $900^{\circ} \mathrm{C}$ can be explained by the different sample weights, as was described by Muwanguzi et al. [10]. The deposited carbon has a tendency to clog the pores. Therefore, the carbon deposition on a surface of the

Table 2. Composition of different layers in sample lump reduced at $900^{\circ} \mathrm{C}$ and flow rate of $0.5 \mathrm{~L} / \mathrm{min}$.

\begin{tabular}{|c|c|c|c|c|c|}
\hline \multirow{4}{*}{\multicolumn{2}{|c|}{ 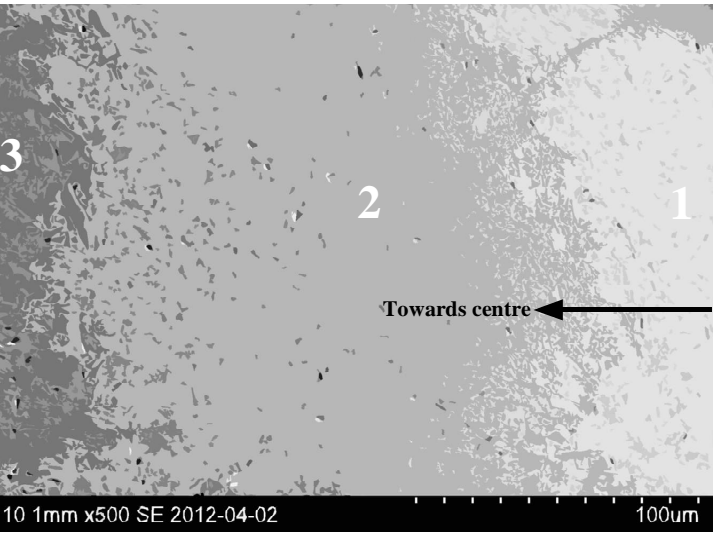 }} & \multirow{4}{*}{\multicolumn{2}{|c|}{$\begin{array}{c}\text { Layer } \\
\\
1 \\
2 \\
3 \\
\end{array}$}} & on (wt\%) & Phase \\
\hline & & & & $95-99$ & $\mathrm{Fe}+(\mathrm{FeO})$ \\
\hline & & & & $80-86$ & $\mathrm{Fe}+\mathrm{FeO}+\left(\mathrm{Fe}_{3} \mathrm{O}_{4}\right)$ \\
\hline & & & & $70-77$ & $\mathrm{Fe}_{2} \mathrm{O}_{3}+\mathrm{Fe}_{3} \mathrm{O}_{4}+\mathrm{FeO}$ \\
\hline
\end{tabular}




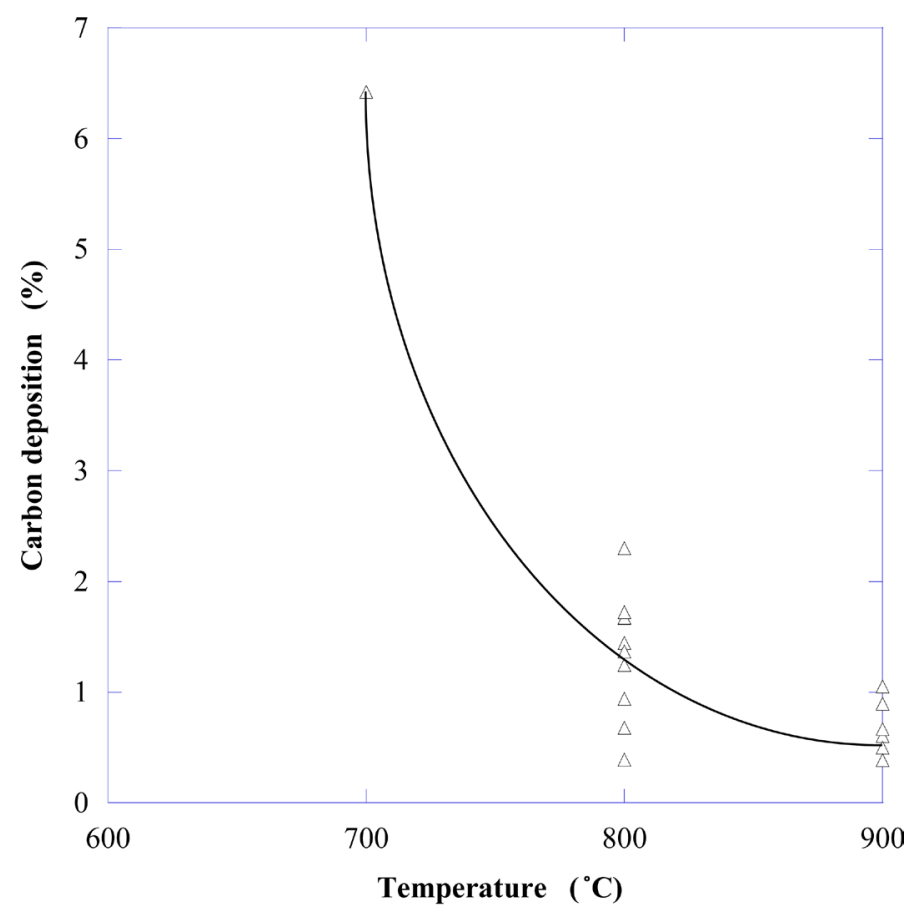

Figure 3. Relationship between the carbon deposition on a sample surface during a reduction experiments and the reduction temperature.

Table 3. Achieved kinetic parameters for direct reduction of natural iron ore at various temperatures.

\begin{tabular}{ccccccccccccc}
\hline $\mathrm{T}$ & Flow & $\mathrm{RD}$ & \multicolumn{4}{c}{$\mathrm{RPM}$} & \multicolumn{3}{c}{$\mathrm{GM}$} & \multicolumn{3}{c}{$\mathrm{VM}$} \\
\hline$\left({ }^{\circ} \mathrm{C}\right)$ & rate $(\mathrm{L} / \mathrm{min})$ & & $\Psi$ & $k_{0}\left(\mathrm{~s}^{-1}\right)$ & $\mathrm{E}_{\mathrm{a}}(\mathrm{kJ} / \mathrm{mol})$ & $\mathrm{r}^{2}$ & $k_{0}\left(\mathrm{~s}^{-1}\right)$ & $\mathrm{E}_{\mathrm{a}}(\mathrm{kJ} / \mathrm{mol})$ & $\mathrm{r}^{2}$ & $k_{0}\left(\mathrm{~s}^{-1}\right)$ & $\mathrm{E}_{\mathrm{a}}\left(\mathrm{kJ} / \mathrm{mol}^{2}\right)$ & $\mathrm{r}^{2}$ \\
\hline 700 & 0.5 & 0.44 & 1.14 & 759 & 111 & 0.993 & 2297 & 120 & 0.986 & 15152 & 132 & 0.962 \\
800 & 0.5 & 0.87 & 1.13 & 614 & 123 & 0.998 & 374 & 119 & 0.997 & 2983 & 133 & 0.930 \\
900 & 0.5 & 0.91 & 3.26 & 631 & 131 & 0.998 & 621 & 128 & 0.995 & 139 & 109 & 0.902 \\
\hline
\end{tabular}

sample inhibited the gas diffusion into the volume of iron oxide grains, resulting in the decreased values of $\mathrm{RR}_{\mathrm{IS}}$ and total reduction degree $\left(\mathrm{RD}_{700}=0.44\right)$ in the experiment at a $700^{\circ} \mathrm{C}$ temperature.

In this study, three models: the volumetric model (VM), the grain model (GM) and the random pore model (RPM) were used for the description of the reduction processes. The main kinetic parameters that were calculated from the different models and the reduction degrees $(\mathrm{RD})$ obtained in the different experiments in this study are listed in Table 3. The relationships between the extent of conversion $(X)$ and time of reduction experiment are shown in Figure 4 for the measured experimental data and for results calculated by using the different models. It can be seen that the VM model predicts significantly higher conversion rates at all temperatures compared to the experimental data and as well as for calculations using the GM and RPM models. This deviation of conversion values $\left(\Delta X_{\mathrm{VM}-\mathrm{EXP}}=X_{\mathrm{VM}}-X_{\mathrm{EXP}}\right)$ increases till maximum values and then decreases during reduction experiments. The significant deviation for the VM model might be due to the fact that the VM model does not take the porosity of 


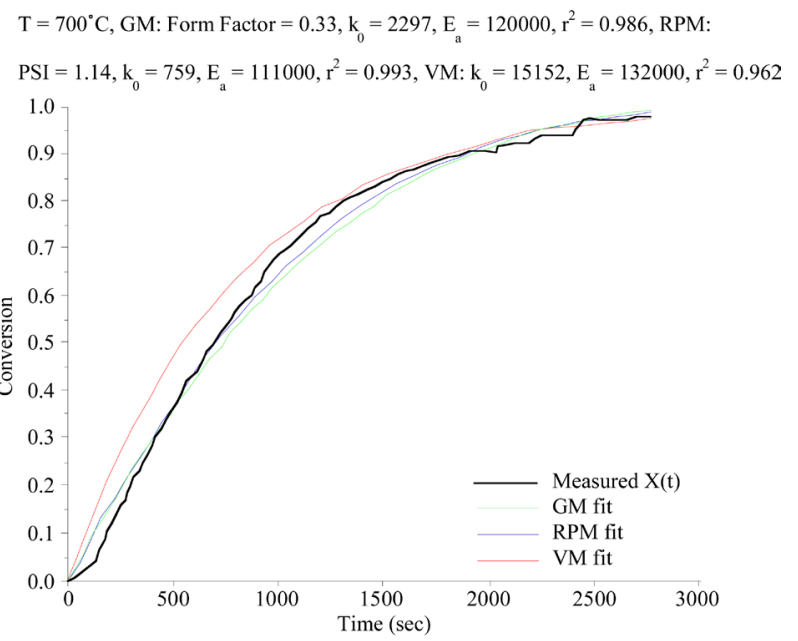

(a)

$\mathrm{T}=800^{\circ} \mathrm{C}, \mathrm{GM}$ : Form Factor $=0.33, \mathrm{k}_{0}=374, \mathrm{E}_{\mathrm{a}}=119040, \mathrm{r}^{2}=0.997, \mathrm{RPM}$ :

PSI $=1.13, \mathrm{k}_{0}=614, \mathrm{E}_{\mathrm{a}}=123000, \mathrm{r}^{2}=0.998, \mathrm{VM}: \mathrm{k}_{0}=2983, \mathrm{E}_{\mathrm{a}}=133000, \mathrm{r}^{2}=0.930$

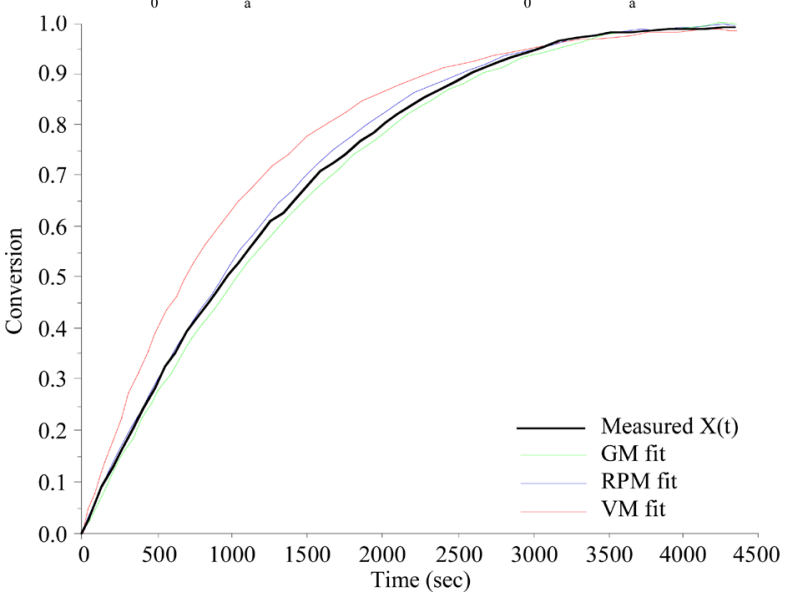

(b)

$\mathrm{T}=900^{\circ} \mathrm{C}, \mathrm{GM}:$ Form Factor $=0.33, \mathrm{k}_{0}=1146, \mathrm{E}_{\mathrm{a}}=134000, \mathrm{r}^{2}=0.995, \mathrm{RPM}$ :

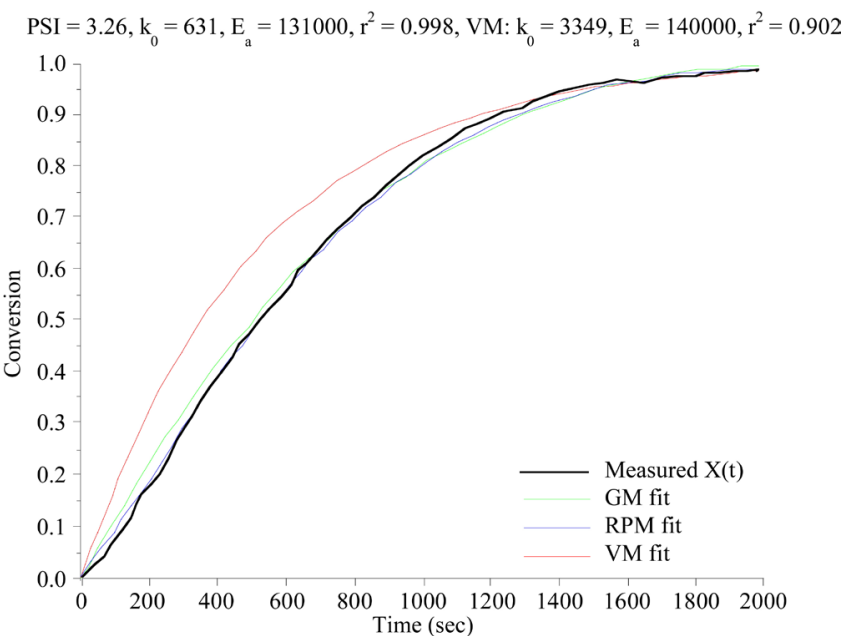

(c)

Figure 4. Approximations of conversion of natural iron ore at the given gas flow rate $(0.5$ L/min) and temperatures: (a) $700^{\circ} \mathrm{C}$; (b) $800^{\circ} \mathrm{C}$; and (c) $900^{\circ} \mathrm{C}$. 
iron oxide sample into consideration. In this model all the reduction potential of the gas is assumed to be concentrated on reducing the core, from surface to centre progressively. Nevertheless, the gas reduction potential decreases as the gas penetrates the iron oxide pores. The apparent porosity of the used natural iron ore was $14 \%$ on average [11]. It should be pointed out that the maximum $\Delta X_{\mathrm{VM}-\mathrm{EXP}}$ values increased with an increased reduction temperature from 0.11 at $700^{\circ} \mathrm{C}$ to 0.16 at $900^{\circ} \mathrm{C}$. This increase of $\Delta X_{\mathrm{VM}-\mathrm{EXP}}$ values may be explained by the higher porosity of the sample at higher experimental temperatures. This is due to the additional formation of internal pores during the reduction of hematite. Thus, the VM model cannot be used for a precise description of the direct reduction kinetics of natural iron ores at given temperatures.

The results obtained from the GM and RPM models, which take porosity into consideration, agreed satisfactorily well with the experimentally measured $X$ values. It can be seen in Figure 4 that the deviation values of $\Delta X_{\mathrm{GM}-\mathrm{EXP}}$ and $\Delta X_{\mathrm{RPM}-\mathrm{EXP}}$ decreased with an increased temperature of the reduction experiments. Furthermore, since the RPM considers the overlapping of the pores and surface area of reduced pores, which actually occurs in the reduction processes, the RPM model produces a better fit with the experimental data than the GM model. This is verified by the highest values of the correlation coefficient $\left(r^{2}=\right.$ 0.993 - 0.998) for calculated RPM relationships, which are given in Table 3. Thus, though the GM model gives close predictions of the iron oxide conversion, the RPM model provides better predictions of the natural iron oxide conversion and thereby the reduction kinetics.

Moreover, in the previous work [8], it was found that the lump samples taken from one block of natural iron ore can have significantly different microstructure and porosity. These differences can drastically influence the reduction rate and time of direct reduction processes. The structural parameter ( $\Psi$ factor) in the RPM model is related to the pore surface of the unreacted sample. Therefore, this model was used in this study to evaluate the effect of the $\Psi$ factor on the reduction processes at different temperatures. According to the data given in $\mathrm{Ta}$ ble 3, the values of $\Psi$ factor were varied in the range from 1 to 4 .

Figure 5 shows the relationships between the extent of conversion $(X)$ and time as a function of the $\Psi$ factor calculated by using the RPM model for reduction experiments at given temperatures. The appropriate values of $k_{0}$ and $E_{a}$ were taken from Table 3 for respective experiments for different temperatures. It is apparent that the parameters of the reduction process such as the conversion extent, reduction rate and reduction time were strongly dependent on the $\Psi$ value in experiments at all given temperatures. For instance, the reduction time for getting value of $X=0.9$ at the $\Psi$ factor of 5 is $1.6-1.8$ times less than that at the $\Psi=1$. It means that the reduction processes for the samples with $\Psi=5$ will be much faster in comparison with that for the samples with $\Psi=1$.

It was found that the maximum disagreement of $X$ values for different $\Psi$ factors corresponds to the reduction time in the range from 800 to 1100 seconds. A 


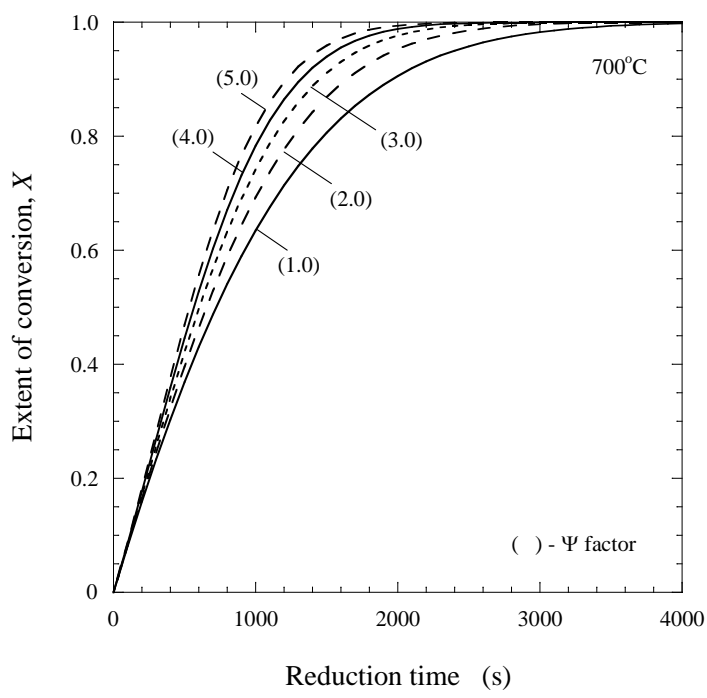

(a)

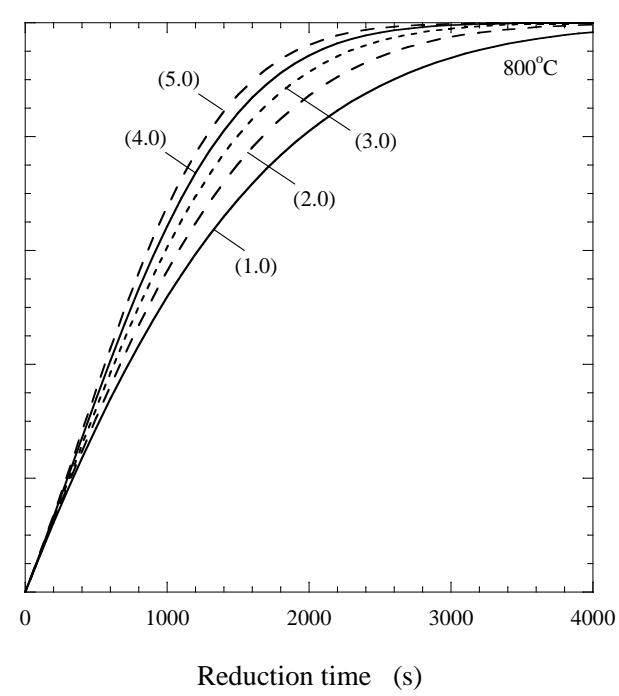

(b)

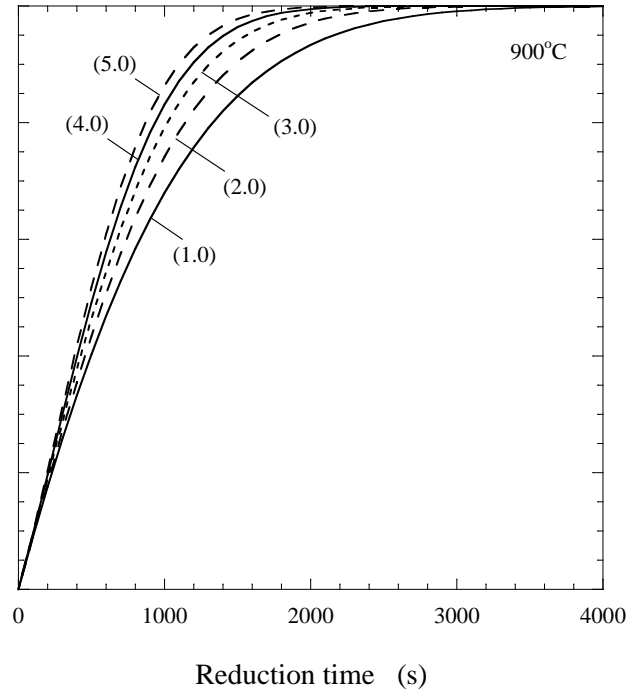

(c)

Figure 5. Relationships between the conversion extent $(X)$ and time as a function of the $\Psi$ factor calculated by using the RPM model for reduction experiments at (a) $700^{\circ} \mathrm{C}$, (b) $800^{\circ} \mathrm{C}$ and (c) $900^{\circ} \mathrm{C}$.

deeper analysis in this range can give a better understanding of the reduction dynamics for given iron oxide structural parameters. Therefore, the conversion extents were calculated by using the RPM model as a function of the $\Psi$ factor for the given gas flow rate $(\mathrm{FR}=0.5 \mathrm{~L} / \mathrm{min})$ and reduction time $(\mathrm{RT}=1000 \mathrm{~s})$. The obtained correlations between the $X$ values and $\Psi$ factor are shown in Figure 6(a) for reduction experiments carried out at different temperatures. It is apparent that the changes of the conversion extents with an increased $\Psi$ factor are almost the same for all temperature experiments. However, the obtained conversion rate at $700^{\circ} \mathrm{C}$ is significantly larger than that at $800^{\circ} \mathrm{C}$. It is due to the fact that the conversion extent does not show the relation with the final reduction degree obtained at different temperatures. Therefore, for an estimation of a temperature effect on the reduction degree at different $\Psi$ factors, a multiplica- 


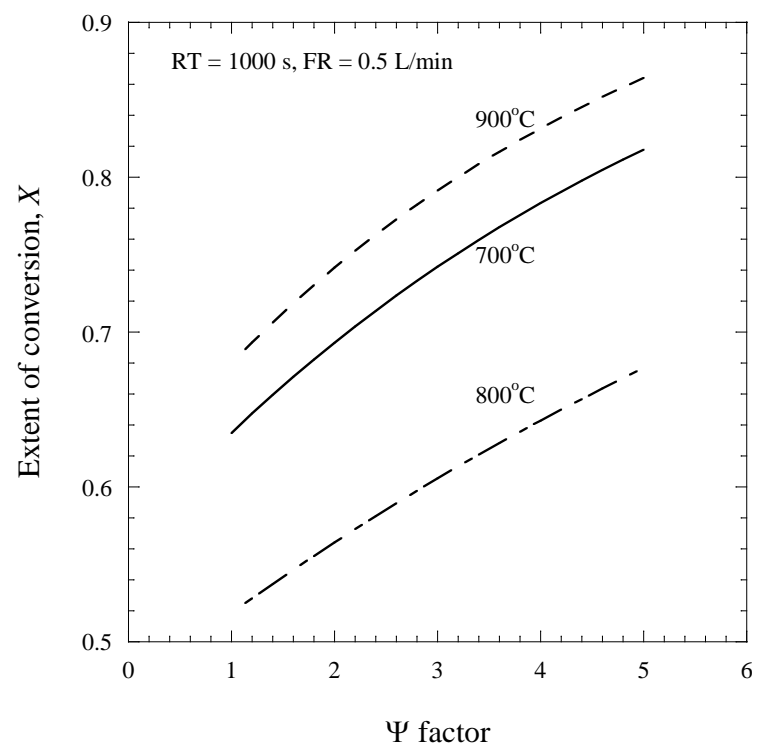

(a)

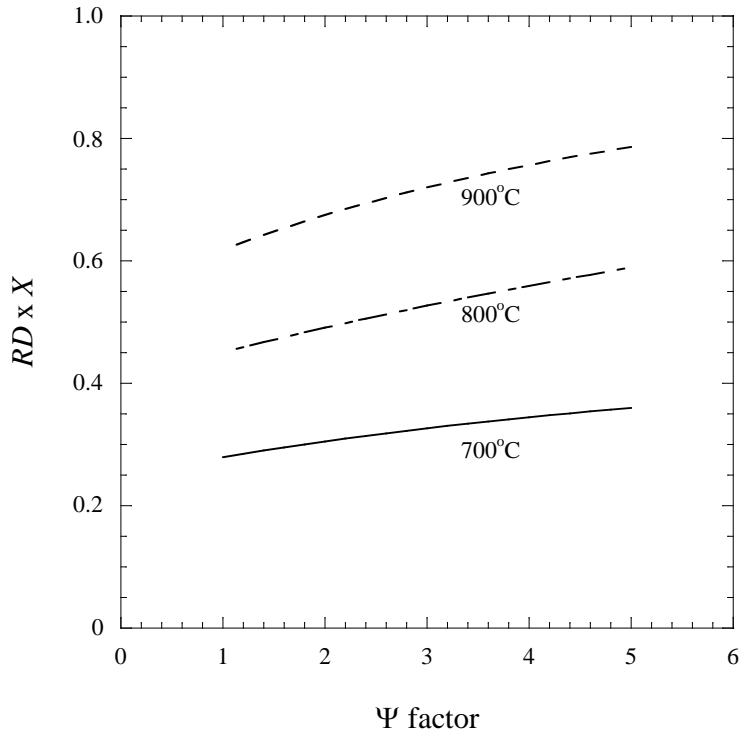

(b)

Figure 6. Effect of the $\Psi$ factor on the (a) conversion extent and (b) the reduction degree value $(\mathrm{RDx} X)$ calculated for different experimental temperatures and a given flow rate $(\mathrm{FR}=0.5 \mathrm{~L} / \mathrm{min})$ and reduction time $(\mathrm{RT}=1000 \mathrm{~s})$.

tion of RD and $X$ values was done and the results are as shown in Figure 6(b). In this case the reduction degree calculated for 1000 seconds of reduction time, increased by $15 \%-22 \%$ with an increase in temperature of $100^{\circ} \mathrm{C}$ in the range from $700^{\circ} \mathrm{C}$ to $900^{\circ} \mathrm{C}$. The RD. $X$ values increased only slightly by $6 \%, 12 \%$ and $14 \%$ at $700,800^{\circ} \mathrm{C}$ and $900^{\circ} \mathrm{C}$, respectively, with an increasing value of $\Psi$ factor from 1 to 5 . Thus, the $\Psi$ factor, which is related to the pore surface in the lump samples, has a significant effect on the reduction rate of the process. This is particularly true for the reduction experiments at higher temperatures.

Therefore, with the RPM model, it can be noted that for known structural pa- 
rameters, $\Psi$, of a given natural hematite ore, solid conversion and reduction extents can be predicted for desired reduction times. Thus, desired reduction parameters can be easily set from the on-set of a reduction process.

Furthermore, as was noted in Figure 2, an effect of the $\Psi$ factor on the conversion extent depends on the reduction time. With the RPM model, the extent of reduction time for given structural parameters can be predicted. To this effect, a difference between $X$ values obtained for the $\Psi$ factor of 1 and 3 ( $\Delta X_{3-1}=X_{3}$ $X_{1}$ ) depending on the reduction time was evaluated. The results are shown in Figure 7(a) for reduction experiments carried out at different temperatures. It can be seen that the $\Delta X_{3-1}$ value obtained in all temperature experiments in-

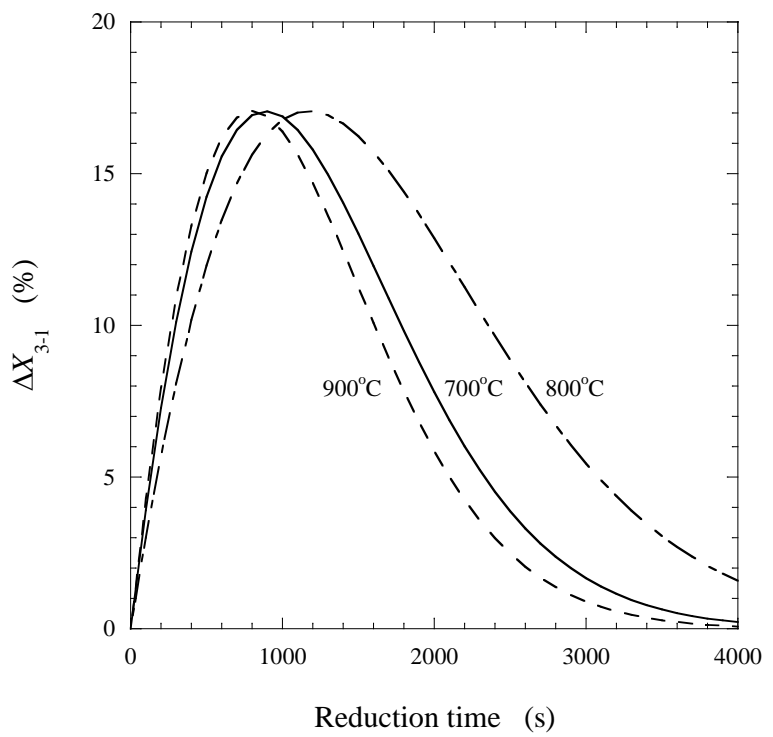

(a)

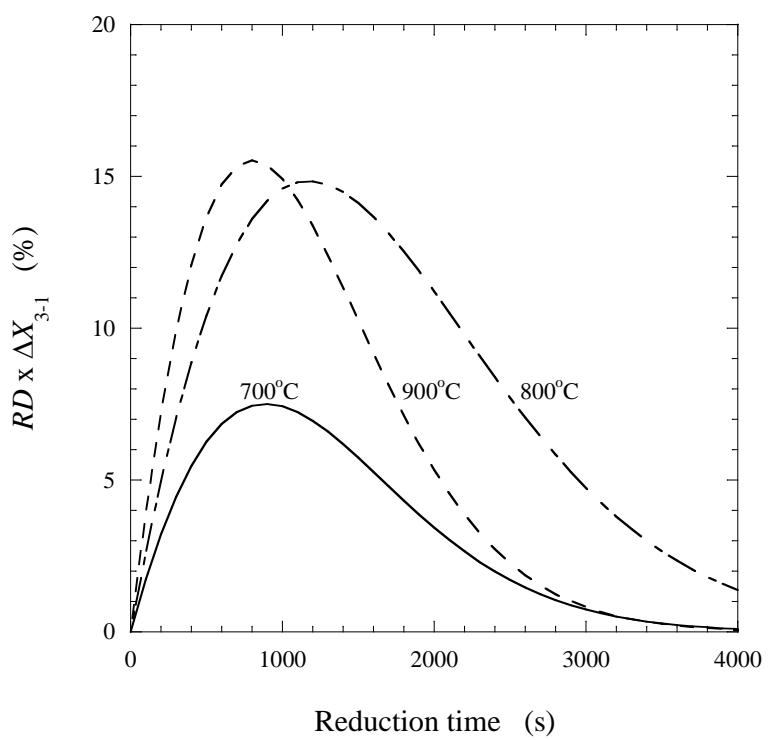

(b)

Figure 7. Changes of the (a) $\Delta X_{3-1}$ and (b) $\mathrm{RDx} \Delta X_{3-1}$ values calculated for the $\Psi$ factor of 1 and 3 depending on the reduction time for different experiment temperatures. 
creases and achieves a maximum value (around 17\%) in the time range from 800 to 1200 seconds. Then, the $\Delta X_{3-1}$ value decreases. However, the maximum $\mathrm{RDx} \Delta X_{3-1}$ values obtained at $700^{\circ} \mathrm{C}, 800^{\circ} \mathrm{C}$ and $900^{\circ} \mathrm{C}$ in the same time range increased on $7.5 \%, 14.5 \%$ and $15.5 \%$, respectively, with an increasing value of $\Psi$ factor from 1 to 3 , as follows from Figure 7(b). Therefore, reduction time has a significant role in achieving desired $\mathrm{RD}$ values for given structural parameters at given temperatures.

The models were applied in the Scilab program for a prediction of the conversion at different temperatures without fixation of the models' kinetic parameters. Such an application of the program and models gives a possibility to elucidate and analyze deviations of the apparent kinetics from the kinetics, which is implied for the case of chemical reactions taking place in the strict conformity to the Arrhenius law. Various physical factors ranging from changing internal conditions in the samples to such external conditions as the temperature, flow rate and concentrations of gasification agents, may contribute to the deviations. An analysis of influence of these factors with respect to the applicability of the models to predict dynamics of raw ore samples' conversion was presented above and is summarized in the conclusion.

\section{Conclusions}

Lumps of natural hematite iron ore from the Muko deposit in Uganda were subjected to direct reduction by a gas mixture of $\mathrm{H}_{2}$ and $\mathrm{CO}\left(\mathrm{H}_{2} / \mathrm{CO}=1.5\right)$ at temperatures of $700^{\circ} \mathrm{C}, 800^{\circ} \mathrm{C}$ and $900^{\circ} \mathrm{C}$ in thermo-gravimetric laboratory experiments. The effect of the reduction temperature on the reduction degree, reduction rate of samples and carbon deposition were investigated. Three models: 1) the Grain Model (GM), 2) the Volumetric Model (VM) and 3) the Random Pore Model (RPM), were used to fit the extents of solid conversion $(X)$. Based on the obtained results, an effect of sample porosity on the reduction parameters was discussed. The following conclusions were obtained:

1) The RPM model provides a better agreement with the obtained experimental results $\left(r^{2}=0.993-0.998\right)$. Furthermore, it provides a better prediction of the natural iron oxide conversion and thereby the reduction kinetics.

2) The structural parameter ( $\Psi$ factor in the RPM model), which is related to the pore structure, was varied in original hematite iron ore samples from 1 to 4 . The $\Psi$ factor has a significant effect on the reduction rate of the process. This is particularly obvious for the reduction experiments at higher temperatures.

3) At the same sample porosity $(\Psi=1)$, the reduction degree can be increased by $15 \%-22 \%$ with a temperature increase of $100^{\circ} \mathrm{C}$ in the temperature range from $700^{\circ} \mathrm{C}$ to $900^{\circ} \mathrm{C}$.

\section{Limitations of this Study}

The study was carried out at a laboratory scale and results obtained are indicative of the expected performance of the natural iron ores at the factory level. On 
the other hand, the parameters of operation at the large scale change and influence behavior differently, which might present different results from those observed at the laboratory scale. These results though can be a starting point of estimation of iron ore behavior and setting of initial operation parameters for large scale operations.

\section{Acknowledgement}

The authors wish to thank the tremendous contributions of Andrey V. Karasev, Assoc. Prof., KTH Royal Institute of Technology, Sweden and Pär G. Jönsson, Prof., KTH Royal Institute of Technology, Sweden plus the Swedish Institute for Development Agency (Sida) that funded this research work.

\section{References}

[1] Turkdogan, E.T. and Vinters, J.V. (1971) Gaseous Reduction of Iron Oxide: Part 1. Reduction of hematite in hydrogen. Metallurgical Transactions, 2, 3175-3188. https://doi.org/10.1007/BF02814970

[2] Turkdogan, E.T. and Vinters, J.V. (1971) Gaseous Reduction of Iron Oxides: Part II. Pore Characteristics of Iron Reduced from Hematite in Hydrogen. Metallurgical Transactions, 2, 3189-3196. https://doi.org/10.1007/BF02814971

[3] Szekely, J. and El-tawil, Y. (1976) The Reduction of Hematite Pellets with Carbon Monoxide-Hydrogen Mixtures. Metallurgical Transactions B, 7, 490-492. https://doi.org/10.1007/BF02652723

[4] Turkdogan, E. and Vinters, J.V. (1972) Gaseous Reduction of Iron Oxides: Part III. Reduction-Oxidation of Porous and Dense Iron Oxides and Iron. Metallurgical Transactions, 3, 1561-1574. https://doi.org/10.1007/BF02643047

[5] Szekely, J. and Evans, J.W. (1970) A Structural Model for Gas-Solid Reactions with a Moving Boundary. Chemical Engineering Science, 25, 1091-1107. https://doi.org/10.1016/0009-2509(70)85053-9

[6] Ishida, M. and Wen, C.Y. (1971) Comparison of Zone-Reaction Model and Unreacted-Core Shrinking Model in Solid-Gas Reactions-I Isothermal Analysis. Chemical Engineering Science, 26, 1031-1041.

[7] Bhatia, S.K. and Perlmutter, D.D. (1980) A Random Pore Model for Fluid-Solid Reactions: I. Isothermal, Kinetic Control. AIChE Journal, 26, 379-386. https://doi.org/10.1002/aic.690260308

[8] Muwanguzi, J.B.A., Yunyun, W., Karasev, V.A., Byaruhanga, K.J. and Pär, G.J. (2013) Investigation of Direct Reduction of Lumps from Natural Hematite Iron Ore. Research and Application of Materials, 1, 73-82.

[9] Nouri, T. and Szekely, J. (1983) The Influence of Carbon Deposition on the Reduction Kinetics of Commercial Grade Hematite Pellets with $\mathrm{CO}, \mathrm{H}_{2}$ and $\mathrm{N}_{2}$. Metallurgical Transactions B, 14, 359-367.

[10] Muwanguzi, A.J.B., Karasev, A.V., Byaruhanga, J.K. and Pär, J. (2014) Effect of Different Factors on Low Temperature Degradation of Hematite Iron Ore Reduction. Metallurgical Research and Technology, 111, 57-66. https://doi.org/10.1051/metal/2014014

[11] Muwanguzi, A.J.B., Karasev, A.V., Byaruhanga, J.K. and Pär, J. (2012) Characterisation of the Physical and Metallurgical Properties of Natural Iron Ore for Iron Production. ISRN Materials Science, 2012, Article ID: 147420. 